\title{
The Method of Solving the Non-Markov`s Problem of the Projects Portfolio Optimization for the Planned Period
}

\author{
Igor V. Kononenko* and Anhelina S. Korchakova \\ National Technical University "Kharkiv Polytechnic Institute", Kyrpychov Str., 02, Kharkiv, 61002, Ukraine
}

Received 8 March 2020; Accepted 29 March 2020

\begin{abstract}
The portfolio optimization is an important phase in projects portfolios management, as it allows achieving the maximum possible results with limited resources. Publications on the project portfolios optimization include both works on portfolio optimization for a specific point in time, i.e. optimization in statics, and papers in the field of portfolio optimization with the account of the projects start times, i.e. optimization in dynamics. In the second case, our special interest is aroused by the works in which the resources receipt and expenditure dynamics in the portfolio is simulated. The aim of the research is to create a method allowing to solve the problem of projects portfolio optimization for the planned period, taking into account the funds receipt and expenditure in time, the previous decisions aftereffect, the projects implementation necessary sequence, and the mandatory inclusion of some projects in the portfolio during the given time period.

Previously, the authors have proposed the three-criterion mathematical model of the projects portfolio optimization for the planned period problem. Target functions there represented the difference between income and expenditure, the risks and social impact of the project portfolio implementation. The problem we consider now appertains to the non-Markov dynamic problems of discrete optimization. To solve it, the special method is needed. It can only be created grounding on the methods for single-criterion problems solving, which also pertain to the type of non-Markov dynamic problems of the discrete optimization.

A method is proposed for solving the problem of projects portfolio optimization for a planned period from the point of view of the difference between revenues and expenses maximization for all projects starting during the planned period. Limitations of the problem require the absence of debts, the abidance by the sequence of project implementation during the whole period of work on the projects, and some projects inclusion in the portfolio during the given period. This task takes into account the aftereffects of previous decisions, i.e. it is a non-Markov dynamic problem of discrete optimization. The proposed method relates to implicit enumeration methods; in the future, it will be used as the basis for the method of a multicriteria problem solving creation.
\end{abstract}

Keywords: projects portfolio; planned period; optimization; non-Markov problem; model; method

\section{Introduction}

Work in the field of projects portfolios optimization originates from studies on the optimal securities portfolios formation [1]. Models of securities portfolios optimization problems usually contain two objective functions and constraints. The first objective function represents the revenue from the portfolio. The second objective function evaluates the risk associated with the portfolio implementation. In [2], risk is assessed using standard deviations for income and fund correlations. Herewith, a number of constraints are taken into account. First, the limit on financing is being considered, including a top and bottom limit. The limit on the funds quantity, the limit on investing in a certain class of assets, the restriction requiring the investment to be a certain minimum value multiple, the limit on operating expenses, and the limit on the proportion between assets are also taken into account [2].

To optimize projects portfolios, researchers used linear, nonlinear, integer, dynamic, stochastic, fuzzy mathematical programming [3].

*E-mail address: igorvkononenko@gmail.com ISSN: 1791-2377 @ 2020 School of Science, HHU. All rights reserved. doi:10.25103/jestr.132.03
Herewith, they considered such objective functions as the overall strategic value of the portfolio, potential portfolio profitability, net present value (NPV), and synergistic effects from the projects included in the portfolio $[4 ; 5]$.

As constraints, the limit on available resources, the limit on logical connections between projects, the ratio of risks, of the project categories, project payback periods were taken into account $[4 ; 5]$.

Quite a lot of researches are devoted to the projects portfolio optimization for a fixed time of their start. Finding the optimal time points for starting projects is not the objective in these works. For instance, the [6] considers the task of the investment projects portfolio formation for the pharmaceutical company. As an objective function, they used the expected value of NPV for all portfolio projects. The constraints in this task included the investment budget and available labor resources. For each project, the input data were the expected value and the standard deviation NPV, the expected value and the standard deviation of the project cost, the need for technical personnel and operators. The value of each project for the company was also set. A total sum of 18 projects was considered that could be included in the portfolio. For the portfolio optimization, the OptQuest software application was applied [7]. This 
program is the Crystal Ball system element and realizes the simulation by the Monte-Carlo method.

The mass of researches emphasize the importance of the project start time determination, which is especially significant from the perspective of the deficit resources distribution $[4 ; 5]$.

The work [8] suggests the mathematical model of the projects for the portfolio choice problem and their start time determination. The objective function in this task equals to the sum of the included in the portfolio projects effects. The simulation contains the row of constraints: the project can be implemented only once, the all-products expenses may not exceed the stated value, the chosen projects have to be finished during the planned period, some projects have to be necessarily included into the portfolio, including the continuing projects. If some project is included into the portfolio, all foregoing projects are to be also included, moreover, they have to be finished before this project's start, but the portfolio can't include projects that are not compatible. The constraints for investment in highly risky or long-term projects may be taken into account by the model. The problem pertains to the Boolean linear programming (01 integer linear programming or 0-1 ILP).

In the research [9] the problem of projects portfolio optimization mathematical model is proposed. It takes into account the projects start time, and its objective function is NPV for the entire portfolio. Constraints take into account the different types of resources availability and the sequence of operations realization in projects.

The [10] regards the problem of the pharmaceutical scientific organization's projects portfolio optimization. There is proposed the model of the problem, in which the objective function reflects the balance in the organization's account at the end of the planned period. This model contains the following constraints: a restriction that takes into account the availability of funds for the projects implementation in all periods, a restriction on the fact that a project can be started no more than once, a limitation of resources, a restriction on the sequence of projects, a restriction on the mandatory inclusion of certain projects in a portfolio, restrictions on the level of debt in each period. The task obtained pertains to the Mixed Integer Linear Programming (MILP) problem. The robust version of this problem is proposed for the case of each project stage undetermined cost.

The [11] suggests the mathematical model and method of project portfolio optimization in the planned period for fuzzy initial data. In the task, the fuzzy part is the objective function, which is counted as the sum of the generalized fuzzy criteria for the projects included in the portfolios. Each project can be started in any year of the planned period. The mathematical model constraints also contain indeterminacy with regard to the planned income, expected profit, the need for investment resources.

In [12] there is proposed the mathematical model of project portfolio optimization in the planned period, taking into account the aftereffects of the previously made decisions. It considers the portfolio of projects, each of which can be started at an arbitrary time during the planned period. In this task, it is proposed to take the number of objective functions into account: the difference between revenues and expenditures in the implementation of the projects portfolio, the risks and the social effect associated with the portfolio implementation. The task model contains the constraints of the funds availability for the portfolio projects implementation, where the funds earned can be spent in the next periods. The related projects implementation sequence is being also taken into account, as well as the necessity of some projects inclusion into the portfolio during the given time period. The task under consideration is the multicriteria non-Markov`s dynamic task of the discrete optimization. "Non-Markov's" are called the optimization problems, where the object state at the $t^{\text {th }}$ stage equals to the function of the state at the previous stage $t-1$ and the controls at the stages $t, t-1, t-2, \ldots, t-p+1$, where $p$ is an integer [13].

For this problem solving, the method from the implicit enumeration methods can be suggested. However, to solve the three-criterion task, it is necessary to have the methods to solve its particular one-criterion tasks.

The analysis of the literature on the project portfolios optimization showed that existing research did not consider methods for solving problems that would take into account the dynamics of funds incoming and expenditure in projects for the planned period and the aftereffects of previous decisions. At that, the tasks should take into account the requirements on the funds earned in projects to a certain period that are to be greater or equal to the expenses for these periods.

The present research objective is to create a method that would solve the problem of the projects portfolio optimization for the planned period, taking into account the receipt and expenditure of funds in time, the previous decisions aftereffects, the necessary sequence of project implementation, the mandatory inclusion of some projects in the portfolio over a given period.

\section{The model of the project portfolio optimization problem}

As a basis, we use the model of the project portfolio optimization for the planned period problem that takes into account the aftereffects, described in [12]. From it, only the first objective function will be left, namely the difference between the revenues and expenses in portfolio.

The problem of the project portfolio optimization is considered. The projects in the portfolio can be started during the time period $[1, \mathrm{~T}]$. In this case, the unit of time will be the period of time to which the start of the project, its completion, funds receipt and expenditure can be attributed. For IT projects, as a unit of time it is convenient to choose one week, sprint duration (2-4 weeks) or 1 month.

Overall number of projects under consideration, that potentially can be included into the portfolio, equals $J$. The $j^{\text {th }}$ project can be started in periods $=\overline{1, T}$, the payment for work from clients can enter in periods $t=\overline{1, T+l^{(J)}-1}$, where $l^{(j)}$ stands for the quantity of the time units, during which the works on $j^{\text {th }}$ project and its financing are undertaken. For the $j^{\text {th }}$ project the client will pay $c_{j r}$ of funds in the $r^{\text {th }}$ period from its realization start, $=\overline{1, l^{(J)}}$.

The expenditure for the $j^{\text {th }}$ project will be equal to $w_{j r}$ in $r^{\text {th }}$ period from its realization start $r=\overline{1, l^{(J)}}$, where the $l^{(j)}$ is the time (quantity of periods) of the project implementation, during which the fund can be spent on it.

Assume that $\max _{j} l^{(j)}=g$.

It is necessary to optimize the project portfolio in such way, that in every period $t=\overline{1, T+g-1}, \quad \forall j=\overline{1, J}$, 
there were enough funds for its implementation, the sequence of related projects implementation was abided by, every project was implemented no more than once, and at the same time the amount of company's profit from the projects implementation was maximum.

$\sum_{t=1}^{T} \sum_{j=1}^{J} \sum_{r=1}^{l^{(j)}}\left(c_{j r}-w_{j r}\right) x_{j t} \rightarrow \max$

$\sum_{k=1}^{t} C_{k}^{0}+$
$\sum_{j=1}^{J} \sum_{p=1}^{t} \sum_{r=1}^{t-p+1} c_{j r} x_{j p} \geq \sum_{j=1}^{J} \sum_{p=1}^{t} \sum_{r=1}^{t-p+1} w_{j r} x_{j p}$

for $t=\overline{1, T}$,

$\sum_{k=1}^{t} C_{k}^{0}+\sum_{j=1}^{J} \sum_{p=1}^{T} \sum_{r=1}^{t-p+1} c_{j r} x_{j p} \geq$

$\sum_{j=1}^{J} \sum_{p=1}^{T} \sum_{r=1}^{t-p+1} w_{j r} x_{j p}$

for $t=\overline{T+1, T+g-1}$,

$\sum_{t=1}^{T} x_{j t} \leq 1, j=\overline{1, J}$,

$x_{j t} \cdot \operatorname{card} P_{j}-\sum_{p \in P_{j}} \sum_{m=1}^{t-l^{(p)}} x_{p m} \leq 0, t=\overline{1, T}$

$\sum_{t=t_{s 1}}^{t_{s 2}} x_{s t}=1, \quad \forall s \in S$,

$x_{j t} \in\{0,1\}, j=\overline{1, J}, t=\overline{1, T}$,

where $C_{k}^{0}, k=\overline{1, T+g-1}$, designates the funds that company is able to put out for the project portfolio realization in the $k$ period. These funds can be negative in case the company needs to gain funds by the portfolio's projects realization in the $r$ period.

$x_{j t}$ is the Boolean variable equaling 1 if the $j^{\text {th }}$ project is started in $t^{\text {th }}$ year, and equaling 0 if opposite.

For the $j$ project the multiplicity of projects numbers $P_{j}$ can be set, which have to be implemented before the $j$ project's start.

The objective function of the (1) task is the difference between revenues and expenditures from all projects starting since the first period to the $T^{\text {th }}$. As the $j^{\text {th }}$ project has been being implemented for $l^{(j)}$ periods, $j=\overline{1, J}$, the objective function (1) accounts the income and expenses for projects, started during periods from the first to $T^{\text {th }}$, even after the $T$ period during $l^{(j)}-1$ periods, $\forall j=\overline{1, J}$.

The constraint (2) requires the funds earned by projects, which have been started in period $t$ and before, in period $t$, $t=\overline{1, T+g-1}$, were more or equal to the expenses for these periods.

The constraint (2) for $t=\overline{1, T}$ applies to the periods when the portfolio's projects implementation can be started.

The constraint (2) for $t=\overline{T+1, T+g-1}$, applies to the periods after $T$, within which there continues the fulfillment of projects started before and during $T$, inclusively.

The first addendum in the left part of the constraint (2) either for the $t=\overline{1, T}$, or for the $t=\overline{T+1, T+g-1}$, i.e.
$F_{1}=\sum_{k=1}^{t} C_{k}^{0}$

are the accumulated to and within the t period funds, which the company can spend on the projects portfolio fulfillment.

The second addendum in the left part of the constraint (2) for $=\overline{1, T}$, i.e.

$F_{2}=\sum_{j=1}^{J} \sum_{p=1}^{t} \sum_{r=1}^{t-p+1} c_{j r} x_{j p}$

are the revenues accumulated to and within the $t$ period from projects, which were started within the periods from the $1^{\text {st }}$ to the $t^{\text {th }}$, inclusively.

The right part of the constraint (2) for $t=\overline{1, T}$

$F_{3}=\sum_{j=1}^{J} \sum_{p=1}^{t} \sum_{r=1}^{t-p+1} w_{j r} x_{j p}$

represents the expenses accumulated to and within the $t$ period from projects which were started during the periods from the $1^{\text {st }}$ to the $t^{\text {th }}$, inclusively.

The second addendum in the left part of the constraint (2) for $t=\overline{T+1, T+g-1}$, i.e.

$F_{4}=\sum_{j=1}^{J} \sum_{p=1}^{T} \sum_{r=1}^{t-p+1} c_{j r} x_{j p}$

are the revenues accumulated to and during the $t$ period from the projects started within periods from the $1^{\text {st }}$ to the $T^{\text {th }}$, inclusively.

The right part of the constraint (2) for $t=$ $\overline{T+1, T+g-1}$, i.e.

$F_{5}=\sum_{j=1}^{J} \sum_{p=1}^{T} \sum_{r=1}^{t-p+1} w_{j r} x_{j p}$

represents the accumulated to and within the $t$ period expenses from projects which were started during the periods from the $1^{\text {st }}$ to the $T^{\text {th }}$, inclusively.

The constraint (3) requires any $j^{\text {th }}$ project, $j=\overline{1, J}$, to be implemented no more than once.

The constraint (4) requires the projects from the $P_{j}$ set to be fulfilled before the $j^{\text {th }}$ project start. The second addendum in (4) is equal to the sum of units, every one of which corresponds the project from the $P_{j}$ set, realized to the time $t$.

Constraint (5) gives the opportunity to set the requirement of the mandatory inclusion the $s^{\text {th }}$ project in the portfolio within the time period $\left[t_{s 1}, t_{s 2}\right], \forall s \in S, S$ - set of projects that must be included in the portfolio.

For simplicity, we do not consider discounting funds over time.

The problem (1)-(6) is the non-Markov`s dynamic task of discrete optimization [Рихтер, 1985]. This problem takes into account the aftereffect of the previously applied controls. So the decision about the start of some $j^{\text {th }}$ project in the period $t$ continuing for $l^{(j)}$ periods will affect the state of the portfolio during the $t+1, t+2, \ldots, t+l^{(j)}-1$ periods.

\section{The projects portfolio optimization method}

Let us consider the method of the $A^{o p t}\left(x_{j t}\right)$ ascertainment. $A^{\text {opt }}\left(x_{j t}\right)$ is the optimal value of the objective function (1) which is achievable in the set of acceptable alternatives. We 
assume that only one project can be started in the period $t, t=\overline{1, T}$.

The method will be given for the case of the objective function minimization, that is why the objective function $A\left(x_{j t}\right)$ will be taken with the minus sign. $r=\overline{1, l^{(j)}}$

1. Set the initial values of the task parameters. As the initial solution, let us take the $x_{j t}=0, j=\overline{1, L}, t=\overline{1, T}$ or the $j(t)=0 \forall t=\overline{1, T}$. Determine $J$ - the number of projects that can be included in the portfolio. We determine the period $T$ for which the portfolio will be formed. We set the income $c_{j r}$ and expenses $w_{j r}$ for each project, as well as the duration $l^{(j)}$ of each project, $j=\overline{1, J}$, .

We determine the funds $C_{k}^{0}$ that the company can allocate for the implementation of the portfolio of projects in the $k$ period, $k=\overline{1, T+g-1}$.

We define the sets $P_{j}, j=\overline{1, J}, S$ and the values of $t_{s 1}$ ,$t_{s 2} \forall s \in S$.

Suppose the $f=0, t^{\prime}=1$, where $t^{\prime}$ marks the present time period. The $f^{*}:=\infty$ will be assigned the objective function top value.

2. Examine the opportunity do not start projects in the $t^{\prime}$ period, i.e. $j=j\left(t^{\prime}\right)=0$.

3. If $t^{\prime}<T$ and $F_{1}+F_{2} \geq F_{3}$ for $t=\overline{1, t^{\prime}}$ and there is no need to start the $s^{\text {th }}$ project necessarily, i.e. $t^{\prime} \neq t_{s 2}$ or $t^{\prime}=t_{s 2}$ and $\exists j(t)=s$ for $t \in\left\{t_{s 1}, \ldots, t_{s 2}-1\right\}, \forall s \in S$, then pass to the section 5. If $t^{\prime}=T$ and $F_{1}+F_{2} \geq F_{3}$ for $t=\overline{1, T}$, $F_{1}+F_{4} \geq F_{5}$ for $t=\overline{T+1, T+g-1}$, and there is no need to start the $s^{\text {th }}$ project necessarily, i.e. $t^{\prime} \neq t_{s 2}$ or $t^{\prime}=t_{s 2}$ and $\exists j(t)=s$ for $t \in\left\{t_{s 1}, \ldots, t_{s 2}-1\right\}, \forall s \in S$, then pass to the section 5 . In other case, we will consider the first project, i.e. $j:=j+1, x_{j t^{\prime}}:=1$.

4. Make the following actions.

4.1 Examine the constraint (2) fulfillment.

If $t^{\prime}<T$ and $F_{1}+F_{2} \geq F_{3}$ for $t=\overline{1, t^{\prime}}$

or if $t^{\prime}=T, \quad F_{1}+F_{2} \geq F_{3}$ for $t=\overline{1, T}, \quad F_{1}+F_{4} \geq F_{5}$ for $t=\overline{T+1, T+g-1}$, then pass to the sub point 4.2 .

If else, pass to the section 7.

4.2 Examine the constraint (3) fulfillment for the $j^{\text {th }}$ project $\sum_{p=1}^{t^{\prime}} x_{j p} \leq 1$

If this fails, pass to the section 7.

4.3 Examine the constraint (4) fulfillment

$$
x_{j t^{\prime}} \operatorname{card} P_{j}-\sum_{p \in P_{j}} \sum_{m=1}^{t^{\prime}-l^{(P)}} x_{p m} \leq 0 .
$$

If this fails, pass to the section 7.
4.4 If $S \neq \varnothing$ and $t^{\prime}=t_{s 2}$ for at least one $s \in S$, we examine the constraint (5) fulfillment.

If $\sum_{t=t_{s 1}}^{t_{s 2}} x_{s t}=1, \forall s \in S$,

then pass to the subsection 4.5.

If this fails, pass to the section 7.

If $S \neq \varnothing$ and $t^{\prime} \neq t_{s 2}, \forall s \in S$, pass to the subsection 4.5.

4.5 If $t^{\prime}=T$, then $D^{\left(t^{\prime}\right)}=0$, pass to the 4.6 subsection. Determine the lower bound for the task objective function within the $T-t^{\prime}$ period. The search has to be made by the assessment of the maximum profit that can be obtained from the completing of projects that were not started yet. The problem constraints are not taken into account here, i.e. consider all the $j \in B_{t^{\prime}}$, where $B_{t^{\prime}}=B \backslash W_{t^{\prime}}$. The $B=\{1,2, \ldots, J\}$ denotes the multitude of projects 'indexes (numbers), which are considered in the task, and the $W_{t^{\prime}}=\left\{j(i), i=\overline{1, t^{\prime}}\right\}$ stands for the multitude of the numbers of projects that are appointed within periods from the $1^{\text {st }}$ to the $t^{\prime}$ th.

Count

$A_{j}^{\left(t^{\prime}\right)}=\sum_{r=1}^{l^{(j)}}\left(c_{j r}-w_{j r}\right) \quad \forall j \in B_{t^{\prime}}$.

Range the $A_{j}^{\left(t^{\prime}\right)}$ from the most to the least. Choose from the ranged values $T-t^{\prime}$ the most ones. Unite the chosen projects` numbers into the $E_{t^{\prime}}$ multitude, then count

$D^{\left(t^{\prime}\right)}=\sum_{\forall j \in E_{t^{\prime}}} A_{j}$

4.6 Under $f-\sum_{r=1}^{l^{(j)}}\left(c_{j r}-w_{j r}\right)-D^{\left(t^{\prime}\right)} \geq f^{*}$ the start of the $j$ th project in $t^{\prime}$ th year unables to find the solution that would be better than the top one. Pass to the section 7 .

4.7 Assign $f:=f-\sum_{r=1}^{l^{(j)}}\left(c_{j r}-w_{j r}\right)$.

5. If $t^{\prime}<T$, consider the next year, $t^{\prime}:=t^{\prime}+1$, pass to the section 2 .

6. Diminish the top value $f^{*}:=f$. Remember the multitude $\overline{W_{T}}=\{j(t), t=\overline{1, T}\}$. If $j\left(t^{\prime}\right) \neq 0$, make the assignation $f:=f+\sum_{r=1}^{l^{(j)}}\left(c_{j r}-w_{j r}\right)$.

7. If $j<L$, assign $j:=j+1, \quad x_{j t^{\prime}}:=1$ and pass to the section 4 .

8. If $t^{\prime}>1$, return one year back, i.e. $t^{\prime}:=t^{\prime}-1$. If $j\left(t^{\prime}\right) \neq 0$, change value $f:=f+\sum_{r=1}^{l^{\left(j\left(t^{\prime}\right)\right)}}\left(c_{j\left(t^{\prime}\right) r}-w_{j\left(t^{\prime}\right) r}\right)$. Pass to the section 7 .

If $t^{\prime}=1$, and $\overline{W_{T}}=\varnothing$, the considered problem has no solutions, else the optimal projects portfolio for the planned period is obtained. 
If $\bar{W}=\{0,0, \ldots, 0\}$, the optimal solution is not to implement any projects in the planned period.

The present method appertains to the methods of the implicit enumeration. If differs from the existing ones by its accounting of the considered task's non-Markov`s character, i.e., it takes into account the aftereffects of the previous decisions.

\section{Conclusion}

The research works in the field of project portfolios optimization are analyzed. The analysis showed that the existing works did not consider methods for solving nonMarkov dynamic problems, i.e. problems that take into account the aftereffects of previous decisions. Herewith, the models should take into account requirements on the funds earned in projects to a certain period to be more or equal to the expenses for these periods.

The present research objective is stated as the creation of the method that allows solving the projects portfolio optimization for the planned period problem. The problem model takes into account the funds receipt and expenditure in time, the previous decisions aftereffects, the necessary sequence of project implementation, the mandatory inclusion of some projects in the portfolio within the given time period.

Previously, the authors proposed a three-criterion mathematical model of the projects portfolio optimization for the planned period problem. Objective functions represented the difference between income and expenses, the risks and social impact of the project portfolio implementation. The problem considered here appertains to non-Markov`s dynamic problems of discrete optimization. To solve it, a special method can be created. In turn, it should ground on methods for single-criterion problems solving, which also belongs to the type of non-Markov's dynamic discrete optimization problems.

There is the method proposed for solving the problem of projects portfolio optimization for a planned period with regard of maximizing the difference between revenues and expenses for all projects starting during the planned period. Constraints of the task require that during the periods of work on projects there were no debts, the necessary sequence of project implementation was abided by, some projects were necessarily included in the portfolio during the given time period. The task takes into account the aftereffects of previous decisions, i.e. is a non-Markov`s dynamic discrete optimization problem. The proposed method pertains to the implicit enumeration methods. To solve problems using this method, a computer program has to be created.

Further it is planned to create a method for solving the multicriteria problem of projects portfolio optimization for the planned period in a non-Markov's setting, grounding on the developed method.

This is an Open Access article distributed under the terms of the Creative Commons Attribution License

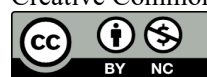

\section{References}

1. H. Markowitz, The Journal of Finance, 7 (1), 77 (1952).

2. K. Liagkouras, $\mathrm{PhD}$ thesis, University of Piraeus (2015).

3. G. Elbok, and A. Berrado, Proceedings of the International Conference on Industrial Engineering and Operations Management, Rabat, Morocco, pp. 21582169 (2017).

4. T. Kremmel, J. Kubalik, and S. Biffl, Applied Soft Computing. 11 (1), 1416 (2011) DOI: 10.1016/j.asoc.2010.04.013

5. P. Morris, and J.K. Pinto, The Wiley guide to project, program, and portfolio management, Hoboken, NJ: J. Wiley \& Sons (2010).

6. E. Vacík, M. Špaček, J. Fotr, and L. Kracík, Ekonomie a Management. 3, XXI, 107 (2018). DOI: 10.15240/tul/001/2018-3-007

7. J. Fotr, and J. Hnilica, Aplikovaná analýza rizika ve finančním management a investičním rozhodování (2nd ed.). Praha: Grada Publishing. (2014).
8. F. Ghasemzadeh, N. Archer, and P. Iyogun, Journal of the Operational Research Society. 50 (7), 745 (1999). https://doi.org/10.1057/palgrave.jors.2600767

9. Y. Shou, and Y. Huang, Journal of Zhejiang UniversitySCIENCE C (Computers \& Electronics). 11(7), 562 (2010). DOI: org/10.1631/jzus.C0910479.

10. F. Hassanzadeh, M. Modarres, H.R. Nemati, and K. Amoako-Gyampah, International Journal of Production Economics, Elsevier, vol. 158(C), 18 (2014). DOI: 10.1016/j.ijpe.2014.07.001

11. И.В. Кононенко, and К.С. Букреева, Управління розвитком складних систем. Збірник наукових праць. 7, 39 (2011).

12. I. Kononenko, A. Korchakova, P. Smolin, Mathematical Modeling and Simulation of Systems. MODS 2019. Advances in Intelligent Systems and Computing, volume 1019. Springer, Cham. 234 (2020). https://doi.org/10.1007/978-3-030-25741-5_23

13. К. Рихтер, Динамические задачи дискретной оптимизации, М.: Радио и связь (1985). 